\title{
Reproductive Behavior of Drosophila melanogaster and $D$. nigrospiracula in the Field and in the Laboratory
}

\author{
Therese Ann Markow \\ Department of Zoology, Arizona State University
}

\begin{abstract}
The reproductive behaviors of two species of fruit fly, Drosophila melanogaster and $D$. nigrospiracula, were compared in field and laboratory populations. A number of differences were observed in the behavior of the two species in their natural habitats. D. melanogaster, which was observed on citrus, mates at its feeding site, whereas $D$. nigrospiracula, which is cactiphilic, mates on a non-resource-based male territory adjacent to its feeding site. In both species large male size is important for reproductive success. However, in $D$. melanogaster smaller males tended to be excluded from the breeding site and were therefore not among the pool of potential mates to which females were exposed. Sex ratios were biased toward females in both species, but the high frequency of female remating in D. nigrospiracula may have provided more mating opportunities for the males of this species. Field observations differed from laboratory observations, and I discuss the importance of these differences for understanding the evolution of Drosophila mating systems.
\end{abstract}

Organisms in natural populations usually exhibit differential reproductive success, because some individuals are more successful in obtaining mates than others. Because differential mating success may influence the genetic composition of subsequent generations, evolutionary biologists are concerned with understanding the mechanisms underlying nonrandom mating (Thornhill \& Alcock, 1983). The relevant mechanisms may be morphologically (Eberhard, 1985) and behaviorally based (Thornhill \& Alcock, 1983).

Drosophila has been a popular organism in empirical studies of the basis of differential mating success. Numerous laboratory investigations of sexual selection in Drosophila have revealed strong correlations of certain traits with male mating success. Among factors reportedly associated with success in Drosophila melanogaster are increased male body size (Ewing, 1961; Markow, 1987; Partridge \& Farquhar, 1983), superior status in aggressive encounters with other males (Dow \& von Schilcher, 1975), the ability to defend a territory, such as patch of food or a drop of feces (Jacobs, 1978), and overall courtship vigor or persistence (Markow, 1987). Many of these traits are associated with male success in other insect species as well (Thornhill \& Alcock, 1983), but the majority of observations on other insects have been made under field conditions, allowing researchers to make evolutionary interpretations with more confidence. Studies in other insect groups have shown that traits associated with mating success differ depending on the ecology of the organisms in question. Although Drosophila mating systems have rarely been studied in natural populations, laboratory findings are unfortunately discussed as though they represent field behav-

This study was supported by National Institutes of Health Grant GM 30638 .

Paul Ankney provided excellent assistance in the field.

Correspondence concerning this article should be addressed to Therese Ann Markow, Department of Zoology, Arizona State University, Tempe, Arizona 85287. ior. Furthermore, findings for $D$. melanogaster, the popular laboratory species, are often generalized to the genus as a whole.

Without direct observation under field conditions, one cannot identify with certainty the factors that cause differential reproductive success in natural populations of Drosophila. I undertook this study of reproductive behavior of Drosophila in natural populations to ask the following questions: (a) Do factors associated with reproductive success in nature correspond closely to what has been observed in the laboratory? (b) Where do Drosophila feeding and reproductive behaviors take place with respect to natural substrates? (c) Do similar factors underlie mating success in species with great differences in their ecology?

The two species discussed in this article, $D$. melanogaster and $D$. nigrospiracula, were chosen for field studies because they represent different subgenera and are characterized by very different ecologies. $D$. melanogaster is a cosmopolitan species associated with man; it oviposits in rotting fruits of many species (Atkinson \& Shorrocks, 1979). D. nigrospiracula is endemic to the Sonoran desert of North America and breeds in necrotic (rotting) saguaro cacti (Carnegia gigantea) in Arizona and Sonora and on cardon (Pachycereus pringlei) in Baja California (Heed, 1978). Extensive descriptions of the reproductive biology of $D$. melanogaster have appeared earlier (Fowler, 1973; Markow, 1987), but only limited information on $D$. nigrospiracula behavior in the laboratory is available (Markow, 1982). In this article I describe the importance of male size and of behavioral traits for mating success in natural populations and compare findings from those populations with results secured under laboratory conditions.

\section{Method}

\section{Studies on Natural Populations}

The natural population of $D$. melanogaster in this study was associated with citrus in southern Tempe, Arizona. When citrus falls, 
it either remains intact and begins internal fermentation, or it cracks open on hitting the ground. Intact pieces do not decay immediately, but they eventually become overgrown with yeast and bacteria. A variety of fruit pieces on which $D$. melanogaster were found covered the ground of the abandoned citrus orchard used in this study: fresh, exposed flesh; undecayed whole fruit with intact skin; and decaying fruit that was moist and covered with microorganisms. The collection sites were approached cautiously to avoid disturbing the flies. A group of investigators approached each site simultaneously to aspirate all the flies present. Flies were aspirated from the three kinds of fruit specimens, separated by sex, and placed into separate containers depending on whether they were single or copulating when collected. An eyepiece micrometer in a Bausch and Lomb dissecting microscope was used to measure males from the different categories of fruit for thorax length, an accurate indicator of body size (Reeve, 1950; Robertson \& Reeve, 1952). The different fruit specimens were examined for eggs and larvae, and the behavioral activities of adults were observed by using a Questar lens. Females were aspirated into separate vials immediately following collection in order to dissect them and look for incidence of insemination. Collections and observations were made at two different times of the year. In Arizona major activity peaks for Drosophila change from the afternoon to morning hours as summer approaches. Therefore, the spring data reported in this article were collected on 4 days in May 1985 from $0730 \mathrm{hr}$ to $0900 \mathrm{hr}$. Data for the winter were collected on 6 days in December 1984 and January 1985 from $1530 \mathrm{hr}$ to $1700 \mathrm{hr}$.

D. nigrospiracula was observed on necrotic saguaro cacti that were located in the desert northeast of Phoenix, Arizona. Necrotic tissue most often appears after an injury such as freezing or other damage to a plant. Streams of exudate run down the plant from the necrotic portion, providing a major site of aggregation of $D$. nigrospiracula. Flies were aspirated from the exudate and from the adjacent, healthy portions of the plants. Adjacent areas included all healthy, external portions of the plant within $500 \mathrm{~cm}$ of the exudate. Flies from both locations on the plants were separated by sex, and their behaviors at each place were noted. The thoraxes of single males and of males in copula were measured, and the data were recorded. As with $D$. melanogaster studies were conducted at two different times of year, December 1984 to January 1985 and May to June 1985. In the winter, collections were made from 0830 to $1000 \mathrm{hr}$ on 5 days and in the spring, from 0630 to $0830 \mathrm{hr}$ on 4 days.

\section{Laboratory Studies of Male Mating Success in $\mathrm{D}$. nigrospiracula}

Unlike $D$. melanogaster, for which exists a large amount of information on mating behavior in the laboratory, $D$. nigrospiracula has received little attention. Experiments were conducted to assess the importance of two factors, male body size and courtship vigor, on mating success of $D$. nigrospiracula males and to make a comparison with laboratory data already available for $D$. melanogaster (Markow, 1987).

Flies were reared on banana medium supplemented with pieces of autoclaved saguaro cactus. Males and females were separated at eclosion and stored until used in mating experiments. Because males take longer to become sexually mature than females (Markow, 1982), they were 10 days old and females were 3 days old when tested. Male mating success was examined by following the methods used with $D$. melanogaster (Markow, 1987). Two males, each marked with a different color of fluorescent dust (U. S. Radium Corporation; see Markow, 1987), were placed in an 8-dram shell vial. A virgin female was introduced after $5 \mathrm{~min}$. Flies were observed for $30 \mathrm{~min}$ or until a mating occurred. The male courting first and the proportion of time each male spent courting were recorded. After copulation both males were anesthetized, and their thorax lengths were determined.
Results

\section{Field Studies}

D. melanogaster. Significantly different sex ratios were found on different types of fruit (Table 1). Although sex ratios, including the total sex ratio, were significantly different from 1:1, fruit types differed in which sex predominated on them. For example, females predominated on the flesh of cracked open pieces, but males were more abundant on the skin of intact fruit. The direction of these sex-ratio differences was identical in both seasons.

The flies were observed engaging in different behaviors on different pieces of fruit. For example, females were observed ovipositing on newly exposed flesh but not on other pieces. The flies, predominantly males, were observed standing still on the outer skin of the unbroken, newly fallen pieces. Copulation was observed only on decaying fruit, where feeding was occurring.

Thorax lengths of males from different fruit types are shown in Table 2. Samples from different days were found to be homogeneous prior to pooling. Body sizes were somewhat larger in the winter collections. Within the feeding and breeding site copulating males did not differ in size from single males at either time of year. However, males on the intact fruit skin were significantly smaller than males at the feeding and breeding sites in both the winter and the spring studies. Some male-male interactions were observed in the form of wing flicking, but these behaviors did not result in one of the participants leaving the immediate location. No uninseminated females were observed at any location.

D. nigrospiracula. At both times of the year, sex ratios differed significantly from 1:1 on all cacti (Table 3). Males predominated on the healthy portions of cactus adjacent to an ooze, but more females were observed on the rotting juice flowing down the plants.

Table 1

Sex Ratio of Drosophila melanogaster Collected on the Flesh of Cracked Open Fruit, Skin of Intact Fruit, and Decaying Pieces of Fruit

\begin{tabular}{|c|c|c|c|c|c|}
\hline \multirow[b]{2}{*}{ Location } & \multicolumn{2}{|c|}{ Male } & \multicolumn{2}{|c|}{ Female } & \multirow[b]{2}{*}{$x^{2}(1: 1)$} \\
\hline & $n$ & $\%$ & $n$ & $\%$ & \\
\hline \multicolumn{6}{|c|}{ Spring } \\
\hline \multirow{4}{*}{$\begin{array}{l}\text { Flesh } \\
\text { Skin } \\
\text { Decaying } \\
\quad \text { fruit }\end{array}$} & 44 & 14.6 & 258 & 85.4 & 151.64 \\
\hline & 207 & 92.0 & 18 & 8.0 & 158.76 \\
\hline & & & & & \\
\hline & 154 & 42.1 & 212 & 57.9 & 9.19 \\
\hline \multirow[t]{2}{*}{ Total } & 405 & 45.4 & 488 & 54.7 & 7.71 \\
\hline & \multicolumn{4}{|c|}{ Winter } & \\
\hline \multirow{4}{*}{$\begin{array}{l}\text { Flesh } \\
\text { Skin } \\
\text { Decaying } \\
\quad \text { fruit }\end{array}$} & 90 & 17.9 & 412 & 82.1 & 206.54 \\
\hline & 253 & 86.1 & 41 & 13.9 & 152.87 \\
\hline & & & & & \\
\hline & 199 & 44.8 & 245 & 55.2 & 4.76 \\
\hline Total & 542 & 43.7 & 698 & 56.3 & 19.63 \\
\hline
\end{tabular}

Note. Data from different days were tested for homogeneity prior to pooling. 
Table 2

Thorax Lengths (in Millimeters) of Male Drosophila melanogaster in a Natural Population

\begin{tabular}{lccc}
\hline $\begin{array}{c}\text { Location or } \\
\text { mating }\end{array}$ & $M \pm S E$ & $n$ & Subset $^{\mathrm{a}}$ \\
\hline & Spring & & \\
& & & \\
Fruit skin & $0.892 \pm 0.006$ & 97 & $\mathrm{a}$ \\
Breeding site & $0.922 \pm 0.006$ & 88 & $\mathrm{~b}$ \\
Mating & $0.910 \pm 0.010$ & 38 & $\mathrm{~b}$ \\
\hline \multicolumn{4}{c}{ Winter } \\
Fruit skin & $0.911 \pm 0.020$ & 99 & \\
Breeding site & $0.959 \pm 0.007$ & 140 & $\mathrm{a}$ \\
Mating & $0.946 \pm 0.009$ & 76 & $\mathrm{~b}$ \\
\hline
\end{tabular}

Note. Data from different days were tested for homogeneity prior to pooling.

${ }^{\mathrm{a}}$ By a Student-Newman-Keuls multiple-range test for unequal samples, two different subsets were found at $\alpha=.05$.

Flies, mostly females, were observed to be feeding actively on the rivers of exudate. Males on the adjacent areas were observed to be fairly uniformly spaced with respect to each other. Individual males were either motionless or walking around in apparently restricted areas. These small areas were usually only $6-7 \mathrm{~cm}$ in diameter. In male-male encounters the flies rushed at each other and flicked their wings until one ran in the other direction. On 3 days in the spring, flies participating in these encounters were observed and aspirated into vials after the encounters were completed. Thirty-one pairs of winners and losers were taken to the laboratory, and their thorax lengths were measured. The thoraxes of winners $(1.22 \pm 0.016 \mathrm{~mm})$ were not significantly larger than those of losers $(1.19 \pm 0.024 \mathrm{~mm})$. Females were courted immediately on arrival at a male's restricted space, and they were observed landing on several different male territories prior to mating. All the mating pairs in this study were collected at sites adjacent to the feeding area with the exception of two pairs collected at the exudate. Flies tended to be larger in the winter collections, but at a given time of year, males that were feeding were the same size as those located in adjacent areas (Table

Table 3

Sex Ratio of Drosophila nigrospiracula Collected on Feeding Sites (Exudate) and Adjacent Areas of Healthy Skin

\begin{tabular}{lrrrrrr}
\hline & \multicolumn{3}{c}{ Male } & & \multicolumn{2}{c}{ Female } \\
\cline { 2 - 3 } Location & $n$ & $\%$ & & $n$ & $\%$ & $\chi^{2}(1: 1)$ \\
\hline \multicolumn{5}{c}{ Spring } \\
Feeding site & 11 & 5.2 & 199 & 94.8 & 168.30 \\
Adjacent skin & 152 & 67.8 & 72 & 32.2 & 28.57 \\
Total & 163 & 37.6 & 271 & 62.4 & 26.87 \\
\hline & 57 & 17.6 & 266 & 82.3 & 135.24 \\
Feeding site & 549 & 73.0 & 92 & 27.0 & 72.28 \\
Adjacent skin & 249 & & & & \\
Total & 306 & 46.1 & 358 & 53.9 & 4.07 \\
\hline
\end{tabular}

Note. Data from different days were tested for homogeneity prior to testing.
Table 4

Thorax Lengths (in Millimeters) of Male Drosophila nigrospiracula in a Natural Population

\begin{tabular}{|c|c|c|c|}
\hline $\begin{array}{l}\text { Location or } \\
\text { mating }\end{array}$ & $M \pm S E$ & $n$ & Subset $^{2}$ \\
\hline \multicolumn{4}{|c|}{ Spring } \\
\hline Exudate & $1.281 \pm 0.04$ & 32 & $\mathbf{a}$ \\
\hline Adjacent skin & $1.294 \pm 0.02$ & 118 & a \\
\hline Mating & $1.354 \pm 0.02$ & 17 & $\mathrm{~b}$ \\
\hline \multicolumn{4}{|c|}{ Winter } \\
\hline Exudate & $1.219 \pm 0.021$ & 11 & $\mathbf{a}$ \\
\hline Adjacent skin & $1.201 \pm 0.022$ & 91 & $\mathbf{a}$ \\
\hline Mating & $1.282 \pm 0.034$ & 65 & $\mathrm{~b}$ \\
\hline
\end{tabular}

Note. Data from different days were tested for homogeneity prior to pooling.

${ }^{a}$ By a Student-Newman-Keuls multiple-range test for unequal samples, two different subsets were found at $\alpha=.05$.

4). At each time of year, mating males were significantly larger than nonmating males.

In $D$. nigrospiracula both the mating and the feeding sites were characterized by the absence of oviposition and of eggs. A systematic search of rotting cacti revealed oviposition sites in flesh that was in the early stages of rotting. These sites were usually accessed by small holes or cracks in the plants.

\section{Laboratory Studies on D. nigrospiracula}

Of 58 pairs of males that were observed, 39 engaged in highly ritualized interactions prior to any behaviors directed toward the females. Males faced each other, with heads touching and fore tarsi locking together. In this position they circled to the left or right (see Figure 1). They did not hit each other with their wings or their legs. Some pairs of males remained in this position for as long as $20 \mathrm{~min}$. These interactions typically ended when the pair separated and walked away from each other. In every case, within a short time one of the males walked over to the female and mounted her. The other male never approached the female. In many cases no court-

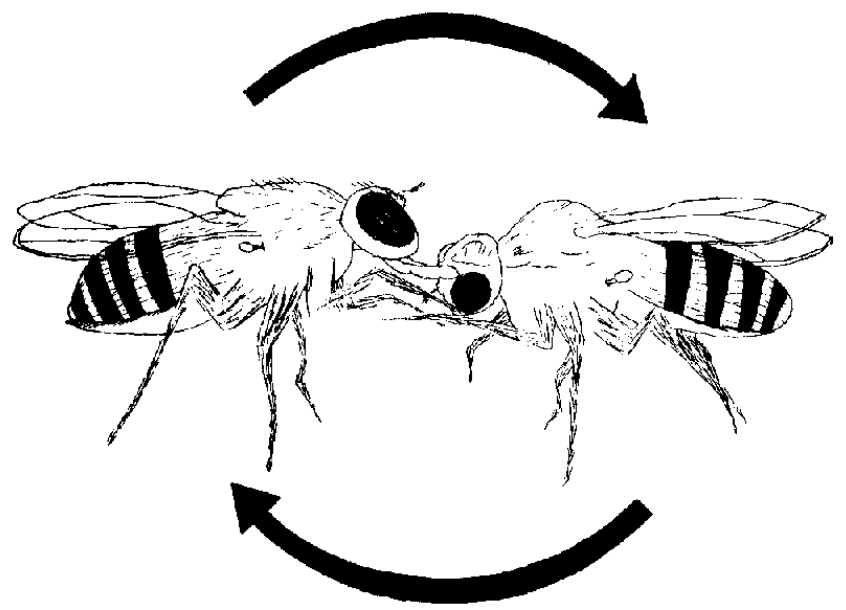

Figure 1. Male-male interaction in Drosophila nigrospiracula. 
ship behaviors, such as wing vibration or licking, preceded copulation. When courtship behavior was exhibited, it was too brief to analyze. In those remaining cases in which males did not engage in the circling behavior with each other $(n=$ 19 ), usually only one male courted, and the brief courtship consisted primarily of licking the female's abdomen. For all 58 pairs, the thoraxes of males that copulated were larger $(1.22 \pm 0.019)$ than those of males that did not $(1.20 \pm 0.021)$, but the difference was not significant. Circling behavior was not observed unless a female was present.

\section{Discussion}

\section{Laboratory and Natural Populations of D. melanogaster}

The first goal of this study was to compare correlates of male success between laboratory and field studies in two species. In the laboratory $D$. melanogaster male success is associated with large body size and courtship vigor (Ewing, 1961; Markow, 1987; Partridge \& Farquhar, 1983). However, in a natural population not all males had equal access to females, and male size was not found to be related to success when only those males at the breeding site were considered. In the laboratory the ability to win in intermale aggressive encounters has been reported to determine subsequent male mating success (Dow \& von Schilcher, 1975). Although this behavior may be the mechanism that excludes smaller males from the feeding and breeding substrate (present study), testing its role was precluded by the absence of any encounters on the citrus in which a clear winner and loser emerged. Partridge, Hoffman, and Jones (1987) also failed to observe any measurable intermale aggression among $D$. melanogaster in a cherry orchard.

Although the present study demonstrates that not all $D$. melanogaster males have equal access to females, it is not known whether all males with access to females mate equally or if males exhibit differential success based on criteria other than size. In the laboratory male success is strongly influenced by a wide range of genetic variables (such as eye color or wing shape) unrelated to size (Bastock, 1956; Bateman, 1948; Boesiger, 1975; Connolly, Burnet, \& Sewell, 1969; Fulker, 1966; Kaul \& Parsons, 1965; Manning, 1961; Markow, 1987; Sturtevant, 1915), any of which may operate in natural populations. Additionally, nongenetic factors such as age and mating experience are important determinants of male success in the laboratory and are likely to influence male success in nature (Long, Markow, \& Yeager, 1980; Markow, Quaid, \& Kerr, 1978). In natural populations of $D$. melanogaster, intrasexual selection seems largely to determine which males have access to females (i.e., are at the breeding site), whereas intersexual selection may play a part in determining success among males that have the chance to court.

\section{Laboratory and Natural Populations of $\mathrm{D}$. nigrospiracula}

Despite a tremendous amount of data that is available on laboratory behavior of $D$. melanogaster and other species, to my knowledge no observations on factors contributing to male success in $D$. nigrospiracula have been reported previously. It is unclear what influence, if any, the curious malemale interaction in $D$. nigrospiracula had on which male subsequently copulated. Copulating laboratory males were no larger than noncopulating males. Although no male-male interactions of this nature were seen on saguaros, the tendency for larger males to be successful in mating was statistically significant in natural populations. It is possible that the circling behavior is fostered or induced by the degree of confinement imposed on the flies by the observation vial and represents an intensified form of the aggressive encounters observed between males on cacti.

In $D$. nigrospiracula females have been observed to land on several different spots resembling male territories prior to mating. It is unknown whether these male territories differ in quality, how many males the females visit prior to copulating, or what sorts of differences males exhibit during courtship that provide for their differential success. However, if larger males are more vigorous courters, female choice for size need not be invoked.

\section{Interspecific Differences in Behavior and Reproduction}

Clearly $D$. melanogaster and $D$. nigrospiracula show contrasting patterns in the way their reproductive behavior occurs with respect to their resources. Males of $D$. melanogaster, frequently in groups, court females on the feeding site. In contrast, $D$. nigrospiracula females fly to non-resource-based male territories where the majority of copulations occur. Neither rotting fruit nor rotting cacti appear to promote the defense of resources by individual males of either species. The relative roles of evolutionary history and recent ecological adaptation in shaping the patterns observed are not yet clear. Resource specificity appears to have been conserved within phylogenetic groups. Species other than melanogaster also use rotting fruit, whereas most species in the repleta group, which includes $D$. nigrospiracula, are cactiphilic. Both species and their relatives may simply be ecologically restricted to substrates that do not allow resource defense.

In both species field matings were found to be nonrandom with respect to male size. However, the disadvantage for small males may arise by different processes in each species. In $D$. melanogaster smaller males are excluded from the feeding and breeding sites. In contrast, $D$. nigrospiracula males found at the feeding sites were no smaller than the males found on territories, and males winning in male-male aggressive encounters were no larger than losers. Nevertheless, in natural populations of $D$. nigrospiracula, the majority of the mating involve larger males on territories.

In both species the total sex ratios of collected flies were female biased. More important than the absolute number of males and females in the population is the operational sex ratio, the number of males and females that are actually capable of mating (Emlen \& Oring, 1977). A number of laboratory findings have revealed biological factors that must influence the operational sex ratio in Drosophila. One of these factors is female receptivity. Under laboratory conditions $D$. 
melanogaster temales may remate after 5-7 days (Markow, 1982 ), but $D$. nigrospiracula females have been observed to mate four times in one morning. Female $D$. nigrospiracula that mated multiply in one morning were also found in nature. Another striking difference between the two species is the age at which males and females become reproductively mature in the laboratory. In $D$. melanogaster both sexes will mate by the time they are 1 or 2 days old. Female $D$. nigrospiracula will mate when they are 3 days old, but males require 9 to 10 days to become sexually mature. This delay in male maturation allows more time for natural selection to act on males prior to mating. Given the high level of female receptivity, the operational sex ratio in $D$. nigrospiracula is probably even more female biased than it appears from the numbers of each sex collected. These observations suggest that $D$. melanogaster males may compete more intensively for matings than $D$. nigrospiracula. An interesting difference between these two species is the more pronounced sex dimorphism in $D$. melanogaster than in $D$. nigrospiracula. In both species males are smaller, but in $D$. melanogaster male and female body coloration is highly dimorphic and males have well-developed sexcombs on their fore tarsi. Sexual dimorphism is one expected outcome of intense sexual selection (Eberhard, 1985).

This study underscores the importance of understanding the breeding system of natural populations prior to generalizing from laboratory data. Nonrandom mating in Drosophila has been popularly attributed to female choice, whereas the above data show clearly that in natural populations of at least one species, certain males are not available for females to choose among because they have been excluded from mating sites.

\section{References}

Atkinson, W., \& Shorrocks, B. (1979). Breeding site specificity in domestic species of Drosophila. Oecologia, 29, 223-232.

Bastock, M. (1956). A gene mutation which changes a behavior pattern. Evolution, 10, 421-439.

Bateman, A. J. (1948). Intrasexual selection in Drosophila. Heredity, 2, 349-368.

Boesiger, E. (1975). The role of sexual selection in the maintenance of genetical heterogeneity of Drosophila populations and its genetic basis. In J. H. F. Abeelen (Ed.), The genetics of behaviors (pp. $167-$ 184). New York: North-Holland.

Connolly, K., Burnet, B., \& Sewell, D. (1969). Selective mating and eye pigmentation: An analysis of the visual component in the courtship behavior of Drosophila melanogaster. Evolution, 23, 548559.

Dow, M. A., \& von Schilcher, F. (1975). Aggression and mating success in Drosophila melanogaster. Nature, 254, 511-512.
Eberhard, W. G. (1985). Sexual selection and animal genitalia. Cambridge, MA: Harvard University Press.

Emlen, S., \& Oring, L. W. (1977). Ecology, sexual selection, and the evolution of mating systems. Science, 197, 215-223.

Ewing, A. (1961). Body size and courtship behavior in D. melanogaster. Animal Behaviour, 9, 93-99.

Fowler, G. (1973). Some aspects of the reproductive biology of Drosophila: Sperm transfer, storage and utilization. Advances in Genetics, 17, 293-360.

Fulker, D. W. (1966). Mating speed in male Drosophila melanogaster. Science, 153, 203-205.

Heed, W. B. (1978). Ecology and genetics of Sonoran desert Drosophila. In P. Brussard (Ed.), Ecological genetics: The interface (pp. 109-126). New York: Springer-Verlag.

Jacobs, M. E. (1978). Influence of $\beta$-alanine on mating behavior and territorialism in Drosophila melanogaster. Behavior Genetics, 8, 487-502.

Kaul, D., \& Parsons, P. (1965). Genotypic control of mating speed and copulation duration in Drosophila. Heredity, 20, 381-392.

Long, C., Markow, T., \& Yeager, P. C. (1980). Relative male age, fertility, and courtship success in Drosophila melanogaster. Behavior Genetics, 10, 163-170.

Manning, A. (1961). The effects of artificial selection for mating speed in Drosophila melanogaster. Animal Behaviour, 9, 82-92.

Markow, T. A. (1982). Mating systems of cactiphilic Drosophila. In J. S. F. Barker \& W. T. Starmer (Eds.), Ecological genetics and evolution: The cactus-yeast-Drosophila model system (pp. 273287). Sydney, Australia: Academic Press.

Markow, T. A. (1987). Genetic and behavioral basis of sexual selection in Drosophila. In M. Huettl (Ed.), The evolutionary genetics of invertebrate behavior (pp. 89-95). New York: Plenum Press.

Markow, T. A., Quaid, M., \& Kerr, S. (1978). Male mating experience and competitive courtship success in Drosophila melanogaster. Nature, 276, 821-822.

Partridge, L., \& Farquhar, M. (1983). Lifetime mating success of male fruit flies (Drosophila melanogaster) is related to their size. Animal Behaviour, 31, 871-877.

Partridge, L., Hoffman, A., \& Jones, J. S. (1987). Male size and mating success in Drosophila melanogaster and Drosophila pseudobscura under field conditions. Animal Behaviour, 35, 468-476.

Reeve, E. C. (1950). Genetical aspects of size allometry. Proceedings of the Royal Society of London, Series B, 137, 515-518.

Robertson, F. W., \& Reeve, E. C. (1952). Studies in quantitative inheritance: $\mathrm{I}$. The effects of selection on wing and thorax lengths in Drosophila melanogaster. Journal of Genetics, 50, 414-448.

Sturtevant, A. (1915). Experiments on sex recognition and the problem of sexual selection in Drosophila. Journal of Animal Behavior, $5,351-366$.

Thornhill, R., \& Alcock, J. (1983). The evolution of insect mating systems. Cambridge, MA: Harvard University Press.

Received July 7, 1987

Revision received December 14, 1987 Accepted January 24, 1988 